\title{
Upper Gastrointestinal bleeding secondary to an enormous hairball
}

\section{Aya Hammami ${ }^{1}$, Sahar Nasr $^{2}$ and Nour Elleuch ${ }^{2}$ \\ ${ }^{1}$ Faculty of medicine of Sousse, University of Sousse, Sousse, Tunisia \\ ${ }^{2}$ Department of Gastroenterology, Hospital Sahloul, Sousse, Tunisia}

A 17- year- old female high school student, with no remarkable medical history, presented to the emergency department with hematemesis and melena. She reported anorexia, weakness and a mild chronic epigastric pain during the last three months, with unrecorded weight loss.

At presentation, the patient was thin. She had no apparent depressive or psychotic symptoms.

Her physical examination showed stable vital signs and a pale skin. Her BMI was $18 \mathrm{Kg} / \mathrm{m}^{2}$ but without clinical signs of nutrient deficiency. Abdominal examination revealed a palpable large,

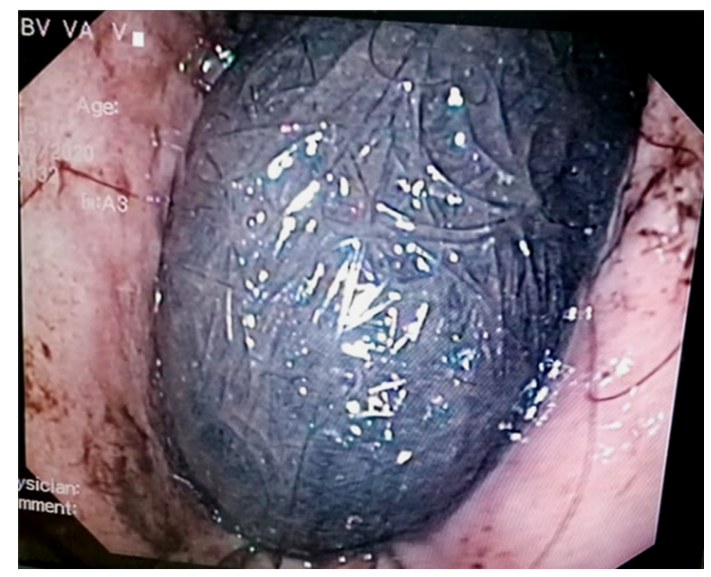

Figure 1. Endoscopic view of the trichobezoar

Copyright: (C2020 Hammami A. This is an open-access article distributed under the terms of the Creative Commons Attribution License, which permits unrestricted use, distribution, and reproduction in any medium, provided the original author and source are credited.

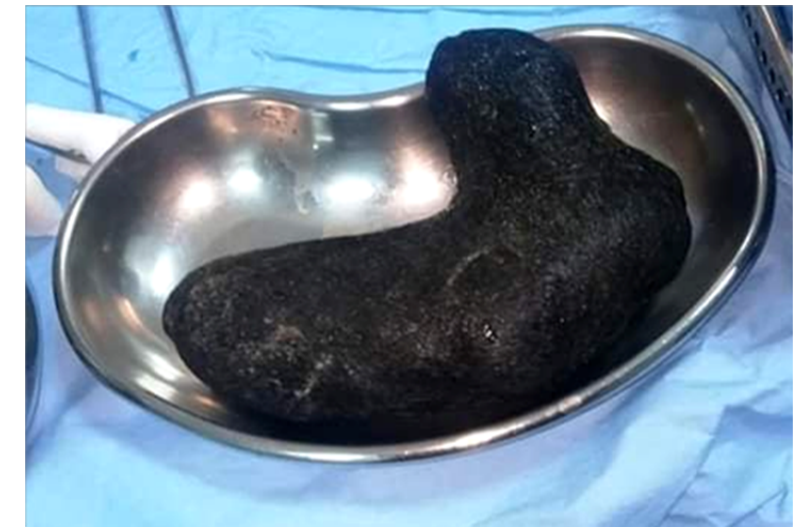

Figure 2. A huge Stomach-shaped hair ball after being extracted from the stomach

mobile, non-tender and ill-defined solid mass in the epigastric and periumbilical region.

The laboratory tests showed hypochromic microcystic anemia with a hemoglobin level of $7.7 \mathrm{~g} / \mathrm{dl}$ and a mean corpuscular volume of $64 \mathrm{fl}$. Renal and hepatic functions were within the reference range.

Upper endoscopy revealed an enormous hairball occupying the whole gastric cavity without active bleeding (Figure 1). A longitudinal gastrostomy was performed and a huge mass, measuring $20 \times 15 \mathrm{~cm}$ and shaped like the stomach, was extracted (Figure 2). The patient discharged home after 6 days, in a good general condition, and was referred to a psychiatrist.
${ }^{\star}$ Correspondence to: Aya Hammami, Faculty of medicine of Sousse, University of Sousse, Sousse, Tunisia, E-mail: aya_med@hotmail.fr, Tel: +21654868578

Received: December 17, 2020; Accepted: December 28, 2020; Published: December 30, 2020 